\title{
In Vivo Placental Transport of Glycine and Leucine in Human Pregnancies
}

\author{
IRENE CETIN, ANNA MARIA MARCONI, ANNA MARIA BAGGLANI, MAURO BUSCAGLIA, \\ GIORGIO PARDI, PAUL V. FENNESSEY, AND FREDERICK C. BATTAGLIA
}

Department of Obstetrics and Gynecology, San Paolo Institute of Biomedical Sciences, University of Milano, Italy [I.C., A.M.M., A.M.B., M.B., G.P.], and Mass Spectrometry Research Resource [P.V.F.] and the Division of Perinatal Medicine, Department of Pediatrics, University of Colorado School of Medicine, Denver, Colorado 80262 [F.C.B.]

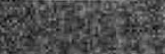

$\mathrm{L}-\left[1-{ }^{13} \mathrm{C}\right]$ Glycine and $\mathrm{L}-\left[1-{ }^{13} \mathrm{C}\right]$ leucine were infused as a bolus into 12 pregnant patients carrying normal fetuses before fetal blood sampling at gestational ages ranging from 20 to $37 \mathrm{wk}$. Maternal venous samples were obtained every $2-3$ min for 15 min after the bolus infusion. Fetal samples were obtained from the umbilical vein within $15 \mathrm{~min}$ of the bolus. Amino acid plasma enrichments (molar percent enrichment) were determined by gas chromatography-mass spectroscopy and their concentrations by ion exchange chromatography. The ratios of glycine and leucine transfer were assessed from fetal/maternal enrichment ratios for each amino acid. We now report that over the gestational age range of $20-37 \mathrm{wk}$, under relatively undisturbed fetomaternal conditions (fetal blood sampling), human placental glycine transfer is limited, with a glycine/leucine ratio $=0.16 \pm 0.02$. We
\end{abstract}

hypothesize that, in human pregnancies, the relative rates of in vivo transplancental transport of amino acids can be assessed indirectly utilizing fetal blood sampling and stable isotope methodology. The application of this approach to leucine and glycine demonstrates that the transfer of leucine is rapid (demonstrable in seconds), whereas that of glycine is more limited. (Pediatr Res 37: 571-575, 1995)
Abbreviations
MPE, molar percent enrichment
FBS, fetal blood sampling
GCMS, gas chromatography-mass spectroscopy
F/M, fetal plasma/maternal plasma

Amino acid transport properties for the human placenta in late gestation have been studied extensively in vitro utilizing two different approaches: isolated microvesicles from either the maternal or basal surfaces $(1,2)$ or the perfused placental lobule or entire placenta $(3,4)$. The properties of the perfused placenta with respect to amino acid transport have been somewhat surprising in that very little difference in placental clearance for amino acids such as glycine and leucine has been found (5).

In contrast, in vivo studies of the ovine placenta in late gestation have shown that glycine and leucine are transported at very different rates (6). These latter observations may reflect species differences which are likely to exist between the human hemochorial placenta and the ovine epitheliochorial placenta

Received July 8, 1994; accepted January 2, 1995.

Correspondence: Frederick C. Battaglia, M.D., University of Colorado Health Sciences Center, 4200 E. Ninth Ave., B-199, Denver, CO 80262.

Reprint requests: Irene Cetin, M.D., Department of Obstetrics and Gynecology, University of Milano, Ospedale San Paolo, Via A. di Rudini, 8, 20142 Milano, Italy.

Supported by the Italian National Research Council (CNR), Targeted Projected "Prevention and Control of Disease Factors," Subproject "FATMA" No. 93.00711.PF41.115.21807, by NATO Grant 88.0191 , and by NIH Center Grant HD20761. with regard to amino acid transport properties. However, it may also reflect, in part, changes in amino acid transport imposed upon the human placenta by the perfusion conditions, notably a relatively low $\mathrm{O}_{2}$ consumption of the placenta. The question of whether permeability studies on the perfused human placenta can provide a reliable description of in vivo characteristics cannot be answered until in vivo data are available for comparison.

Despite the importance of this subject to human fetal nutrition, in vivo properties for maternal-fetal transport of amino acids across the human placenta have not been characterized, in part for technical reasons. To measure placental transport in vivo would require measurements of umbilical blood flow and arterial-venous differences of substrate tracer and tracee concentrations across the umbilical circulation. This is feasible at the time of delivery, but not during fetal life before delivery. Work in animals has clearly demonstrated striking differences in arterial-venous concentration differences of amino acids determined during fetal life versus data obtained at the time of delivery. Thus, studies at cesarean section or at the time of vaginal delivery could be misleading in terms of providing a description of the normal fetal and placental environment. 
Until recently, it was not even possible in human pregnancies to study the appearance of a labeled compound derived from the maternal circulation in the fetal circulation under conditions in which the fetus was not delivered. However, with the advent of FBS, it is now possible to collect fetal blood at different gestational ages without interruption of pregnancy (7-9). Thus, it is now possible to measure the appearance of a tracer amino acid in the fetal circulation that has been infused into the maternal circulation. Such information in the relative rate of appearance of a tracer amino acid in the fetal circulation will be a complex function of rates of amino acid appearance within the placenta and within the fetus and also of placental permeability.

The present study addresses the relative rates of appearance of tracer glycine and leucine with the goal of assessing whether an approach which utilizes a bolus maternal infusion and FBS can assess whether amino acids belonging to different transport systems cross the placenta at different transport rates. The study utilizes non-steady state stable isotope methodology to examine the appearance of $\mathrm{L}-\left[1-{ }^{13} \mathrm{C}\right]$ glycine and $\mathrm{L}-[1-$ ${ }^{13} \mathrm{C}$ ]leucine in the fetal umbilical circulation following a bolus infusion into the maternal circulation. FBS as first described by Daffos et al. (10) which we have modified (9) was performed under ultrasonic guidance.

\section{METHODS}

Informed consent was obtained from all patients. The protocols were approved by the San Paolo Institute Board which has the responsibility for evaluation of all submitted scientific studies and were also approved by the University of Colorado School of Medicine Human Subjects Committee. The procedures were performed in the Department of Obstetrics and Gynecology of the San Paolo Institute of Biomedical Sciences.

Patients. Twelve patients were studied between 20.1 and $37.1 \mathrm{wk}$ of gestation at the time of FBS. FBS was performed under ultrasonic guidance and fetal blood was obtained from the umbilical vein as described by Marconi et al. (9). The site of sampling was assessed by ultrasonographic imaging with a $5-\mathrm{MHz}$ sector transducer, by determination of the nonpulsatile flow in the vessel, and by imaging the direction of flow by observing the bubbling effect produced by the injection of 2 $\mathrm{mL}$ of isotonic saline solution to verify the venous site of sampling during the procedure. The reliability of fetal samples was also assessed by comparison of maternal and fetal sample hematocrit and hemoglobin concentrations and by analysis of red cell volumes with a Coulter Counter (Coulter Electronics, Hialeah, FL).

Table 1 summarizes the clinical information for the study patients, and presents the indications for FBS. All pregnancies were normal and resulted in the delivery of a normal term appropriately grown infant according to Italian birth weightgestational age standards (11).

Protocol of the study. $\mathrm{L}-\left[1{ }^{13} \mathrm{C}\right]$ Glycine and $\mathrm{L}-\left[1-{ }^{13} \mathrm{C}\right]$ leucine (99\% purity) were obtained from Isotec Inc. (Miamisburg, OH) and from MSD Isotopes (Cambridge, Mass), respectively. Ninety-nine percent purity was confirmed by GCMS analysis in our laboratory. Sterile solutions for i.v. use were prepared
Table 1. Clinical information for the patients

\begin{tabular}{|c|c|c|c|c|}
\hline $\begin{array}{c}\text { Case } \\
\text { no. }\end{array}$ & $\begin{array}{c}\text { Maternal } \\
\text { age (y) }\end{array}$ & $\begin{array}{c}\text { Maternal } \\
\text { weight } \\
(\mathrm{kg})\end{array}$ & $\begin{array}{c}\text { Gestational } \\
\text { age (wk) }\end{array}$ & Indications \\
\hline 1 & 23 & 83 & 20.1 & Seroconversion toxoplasmosis \\
\hline 2 & 30 & 60 & 23.3 & Seroconversion toxoplasmosis \\
\hline 3 & 30 & 65 & 22.1 & Maternal chicken pox \\
\hline 4 & 26 & 58 & 36.2 & Maternal thrombocytopenia \\
\hline 5 & 26 & 53 & 21.3 & Seroconversion toxoplasmosis \\
\hline 6 & 25 & 82 & 23.1 & Seroconversion toxoplasmosis \\
\hline 7 & 21 & 48 & 22.1 & Seroconversion toxoplasmosis \\
\hline 8 & 37 & 72 & 21.0 & Seroconversion toxoplasmosis \\
\hline 9 & 29 & 78 & 36.0 & Maternal thrombocytopenia \\
\hline 10 & 36 & 73 & 37.1 & Maternal thrombocytopenia \\
\hline 11 & 26 & 64 & 21.5 & Seroconversion toxoplasmosis \\
\hline 12 & 34 & 69 & 32 & Maternal thrombocytopenia \\
\hline
\end{tabular}

with $56 \mathrm{mg}$ of $\mathrm{L}-\left[1-{ }^{13} \mathrm{C}\right]$ glycine and $70 \mathrm{mg}$ of $\mathrm{L}-\left[1-{ }^{13} \mathrm{C}\right]$ leucine dissolved in $10 \mathrm{~mL}$ of isotonic saline solutions.

The studies were performed after an overnight fast of at least $10 \mathrm{~h}$. The patients were in the supine position at the time of study. A catheter was placed in the left brachial vein, and a time zero sample was taken followed by a 30 -s infusion of the tracer solution sufficient to provide $0.8 \mathrm{mg} / \mathrm{kg}\left[{ }^{13} \mathrm{C}\right]$ glycine and $1.0 \mathrm{mg} / \mathrm{kg}\left[{ }^{13} \mathrm{C}\right]$ leucine. Over the $15 \mathrm{~min}$ after the primed infusion, three to four maternal brachial venous blood samples were collected to ensure that maternal samples were obtained bracketing the single fetal sampling.

A FBS of $1 \mathrm{~mL}$ fetal blood was obtained from the umbilical vein. Inasmuch as there have been no previous studies using tracer methodology and FBS, we attempted to determine whether the data within the same patient were changing from moment to moment. For this reason, in four cases, a fetal sample was taken before and after obtaining the sample required for clinical service, and the results were compared.

Both maternal and fetal samples were centrifuged immediately, and the plasmas stored in liquid $\mathrm{N}_{2}$ until analysis.

Analytical methods. Plasma leucine and glycine enrichments were determined by GCMS using techniques described previously $(12,13)$. Tert-butyldimethylsiloxy derivatives of glycine and leucine were prepared and analyzed in triplicate by GCMS, and the mean value was used in all further calculations. Peaks for $\left[{ }^{12} \mathrm{C}\right]$ - and $\left[{ }^{13} \mathrm{C}\right]$ glycine were recorded at 246 and 247 $\mathrm{m} / \mathrm{z}$, respectively. For leucine, the recordings were made at 302 and $303 \mathrm{~m} / \mathrm{z}$, respectively. Plasma enrichments (MPE) of glycine and leucine were calculated using the difference in peak area ratios between unenriched and enriched samples. Amino acid concentrations were determined on a JEOL (Tokyo, Japan) amino acid analyzer by ion-exchange chromatography using norleucine as an internal standard. For Figure 2, tracer concentrations of leucine and glycine were calculated from the concentrations and enrichments as:

$$
\begin{aligned}
\text { Tracer concentration } & =\text { conc } \times(\mathrm{MPE}) \times 0.01 \\
\text { Tracee concentration } & =\text { conc }- \text { tracer }
\end{aligned}
$$

where conc $=$ total concentration determined by amino acid chromatography.

As an assessment that the fetuses were in a normal metabolic state, $\mathrm{O}_{2}$ content, $\mathrm{pH}$, and $\mathrm{pO}_{2}$ were measured with a radiom- 
Table 2. FBS plasma leucine and glycine enrichments and concentrations

\begin{tabular}{|c|c|c|c|c|c|c|c|c|c|}
\hline \multirow[b]{2}{*}{ Case no. } & \multirow{2}{*}{$\begin{array}{l}\text { Time sample } \\
\text { (s) }\end{array}$} & \multicolumn{4}{|c|}{ Enrichments (MPE \%) } & \multicolumn{4}{|c|}{ Concentrations $(\mu \mathrm{mol} / \mathrm{ml})$} \\
\hline & & M Gly & F Gly & M Leu & F Leu & M Gly & F Gly & M Leu & F Leu \\
\hline 1 & 40 & 57.0 & 1.1 & 51.2 & 13.0 & 0.382 & 0.233 & 0.231 & 0.182 \\
\hline $2 F_{1}$ & 50 & 26.0 & 2.2 & 31.5 & 20.8 & 0.246 & 0.275 & 0.131 & 0.198 \\
\hline $2 \mathrm{~F}_{2}$ & 60 & 27.0 & 2.8 & 31.0 & 22.7 & 0.267 & 0.234 & 0.137 & 0.192 \\
\hline 3 & 67 & 16.7 & 0.4 & 22.5 & 5.1 & 0.251 & 0.251 & 0.098 & 0.139 \\
\hline 4 & 152 & 27.6 & 3.0 & 28.5 & 15.4 & 0.271 & 0.288 & 0.123 & 0.141 \\
\hline 5 & 255 & 38.7 & 7.5 & 23.5 & 23.7 & 0.143 & 0.283 & 0.114 & 0.220 \\
\hline 6 & 364 & 23.7 & 3.2 & 13.9 & 13.4 & 0.160 & 0.167 & 0.112 & 0.162 \\
\hline 7 & 465 & 33.8 & 4.4 & 24.2 & 18.8 & 0.147 & 0.176 & 0.121 & 0.227 \\
\hline 8 & 917 & 13.7 & 2.7 & 13.2 & 11.0 & 0.159 & 0.178 & 0.101 & 0.164 \\
\hline $9 F_{1}$ & 390 & 7.7 & 1.7 & 10.0 & 9.7 & 0.163 & 0.238 & 0.100 & 0.160 \\
\hline $9 \mathrm{~F}_{2}$ & 400 & 7.4 & 1.31 & 9.9 & 9.3 & 0.163 & 0.197 & 0.100 & 0.142 \\
\hline $10 F_{1}$ & 199 & 27.9 & 3.4 & 25.2 & 18.8 & 0.127 & 0.237 & 0.123 & 0.198 \\
\hline $10 \mathrm{~F}_{2}$ & 228 & 25.5 & 3.0 & 22.8 & 10.5 & 0.121 & 0.200 & 0.121 & 0.198 \\
\hline 11 & 290 & & & 7.0 & 6.2 & & & & \\
\hline $12 \mathrm{~F}_{1}$ & 279 & & & 9.3 & 5.8 & 0.152 & 0.178 & 0.108 & 0.108 \\
\hline $12 F_{2}$ & 300 & & & 8.7 & 5.1 & 0.152 & 0.178 & 0.108 & 0.108 \\
\hline
\end{tabular}

The data for the plasma concentrations of leucine and glycine and for the plasma enrichments of leucine and glycine are presented for all fetal samples. In four patients two fetal samples were obtained, and the data are presented as $F_{1}$ and $F_{2}$. The maternal data represent data for the sample obtained simultaneously with the fetal sample or extrapolated from the two maternal data points that bracketed the fetal sample.

eter ABL-330 analyzer (Radiometer Co., Copenhagen, Denmark) and compared to previously published data from our laboratory (14).

Statistical analysis. Data are usually described as mean \pm SE. Significance of the difference between groups was tested by the Mann-Whitney test. The data for the amino acid enrichments and their ratios were analyzed as a function of time by nonlinear regression analysis using a SAS Proc NLIN program. The significance of the difference for the glycine and leucine curves was tested with a Wald test (15).

\section{RESULTS}

Table 2 presents the maternal and fetal glycine and leucine concentrations and their plasma enrichments for all patients. The times the fetal samples were obtained ranged from 40 to $917 \mathrm{~s}$ with $\mathrm{t}=0$ being the time of bolus infusion. A wide range of times from the bolus injection to the fetal sampling permitted analysis of the data for calculation of nonlinear regression equations. These equations were as follows:

Leucine: $(\mathrm{F} / \mathrm{M})^{\mathrm{MPE}}=(0.94 * \mathrm{t}) /(60.6+\mathrm{t}) \pm 0.191(n=16)$

Glycine: $(\mathrm{F} / \mathrm{M})^{\mathrm{MPE}}=(0.23 * \mathrm{t}) /(153.4+\mathrm{t}) \pm 0.036(n=13)$

The regression lines and 95\% confidence limits are presented in Figure 1. The difference between these curves was significant $(p<0.0001)$. A similar analysis was done for the ratio of fetal/maternal L- $\left[1-{ }^{13} \mathrm{C}\right]$ leucine and glycine concentrations. Figure 2 presents the regression lines and 95\% confidence limits for these data. The equations for the regressions were:

$$
\begin{aligned}
& \text { Leucine: }(\mathrm{F} / \mathrm{M})=(0.99 \mathrm{t}) /(94.7+\mathrm{t}) \pm 0.201(n=15) \\
& \text { Glycine: }(\mathrm{F} / \mathrm{M})=(0.23 \mathrm{t}) /(239.7+\mathrm{t}) \pm 0.037(n=13)
\end{aligned}
$$

Two fetal samples drawn at the beginning and end of the cordocentesis were obtained in patients $2,9,10$, and 12 . The

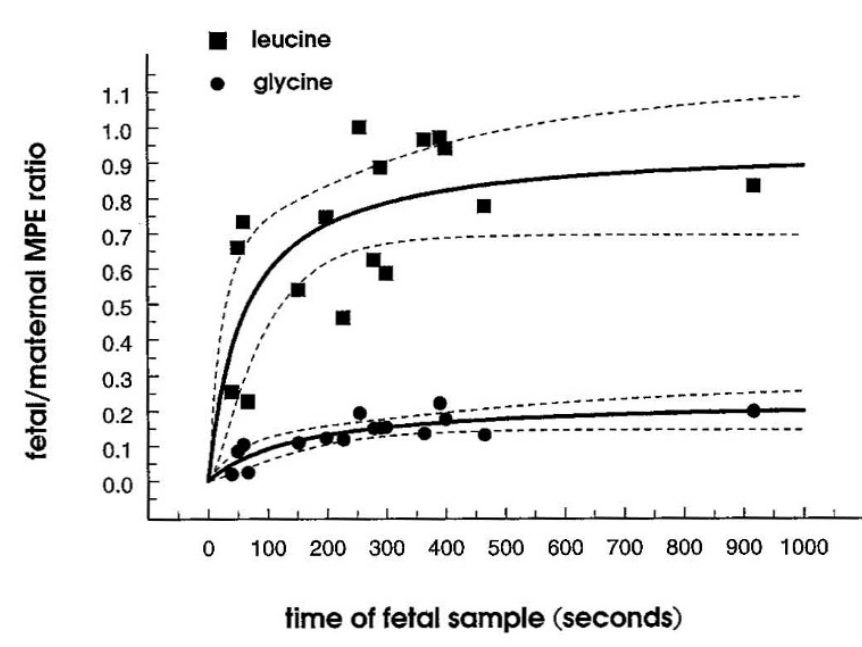

Figure 1. Fetal/maternal enrichment ratio for leucine and glycine $v s$ time of fetal sampling. Nonlinear regression lines and their $95 \%$ confidence limits depicted are: leucine $(\mathrm{F} / \mathrm{M})^{\mathrm{MPE}}=0.94 \mathrm{t} /(60.6+\mathrm{t}) \pm 0.191$; glycine $(\mathrm{F} / \mathrm{M})^{\mathrm{MPE}}$ $=0.23 \mathrm{t} /(153.4+\mathrm{t}) \pm 0.036$. These curves were significantly different $(p<$ 0.0001 , Wald test).

data are presented separately in Table 2 to demonstrate the agreement between two fetal samples in the same patient. The four additional data points were included in the nonlinear regression equations. To test whether the enrichment ratio was influenced by the time of fetal sampling in relation to the bolus infusion, the $\mathrm{F} / \mathrm{M}$ enrichment ratios for leucine and glycine were analyzed as follows: the difference between F/M enrichment ratios of leucine and glycine were calculated from the two regression equations given above. From 300 to $1000 \mathrm{~s}$ the difference changed $<10 \%$ (difference at $300=0.63$, at $1000 \mathrm{~s}$ $=0.69$ ). For all points after $300 \mathrm{~s}$, the $\mathrm{F} / \mathrm{M}$ ratio for leucine was significantly higher $(p<0.0001)$ than that for glycine. Figure 2 presents the $\mathrm{F} / \mathrm{M}$ ratios of tracer/tracee for leucine and glycine analyzed in the same manner. The $\mathrm{F} / \mathrm{M}$ ratio of leucine was significantly higher $(p<0.0001)$ than for glycine. 


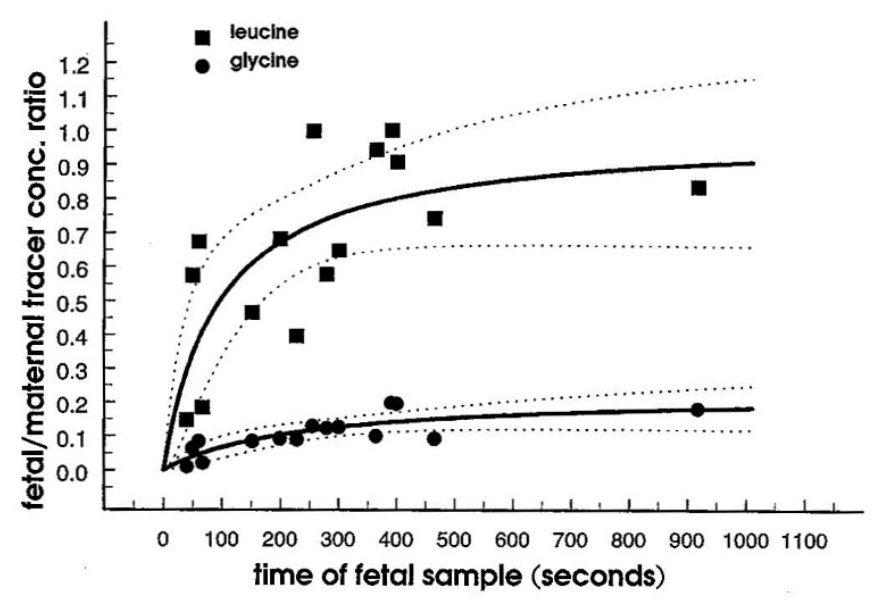

Figure 2. Fetal/maternal tracer concentration ratio for leucine and glycine versus time of fetal sampling. Nonlinear regression lines and their $95 \%$ confidence limits are depicted: leucine $(\mathrm{F} / \mathrm{M})$ tracer concentration $=(0.99 \mathrm{t}) /$ $(94.7+t) \pm 0.201 ;$ glycine $(F / M)$ tracer concentration $=(0.23 t) /(239.7+t)$ \pm 0.037 .

\section{DISCUSSION}

A rather extensive literature has developed around in vitro studies of amino acid transport in the human placenta, either utilizing microvesicle preparations prepared from the basal or maternal surfaces of the trophoblast, or utilizing the perfused placenta in vitro (1-4). Studies of the perfused placenta have not shown any marked differences in transport of glycine and leucine. In particular, in the perfused placenta virtually equal placental clearances were reported for glycine and leucine (35.2 \pm 4.1 versus $33.0 \pm 6.8$ clearance index) (5).

Transplacental transport and metabolism of leucine and glycine have been studied in some detail in vivo for the ovine fetus and placenta $(12,13,16,17)$. The traditional view of the transplacental transport of an amino acid refers to the overall process by which an amino acid present in the maternal circulation may be transported across the placenta into the fetal circulation. However, an amino acid entering the fetal circulation from the placenta may not have originated in the maternal circulation, but instead may have been synthesized within the placenta from other precursors. Alternatively, an amino acid may enter from the maternal circulation and be utilized almost entirely within the placenta. In the present study, leucine has been chosen because of its importance as an essential amino acid, although even leucine could in theory be synthesized within the placenta from $\alpha$-ketoisocaproic acid, taken up from the maternal circulation. Studies performed upon pregnant sheep in our laboratory have shown a large entry of glycine from the placenta into the fetal circulation, second only to glutamine in its magnitude (18). However, there is no significant net uptake of glycine from the maternal circulation into the uterus $(19,20)$. Taken together, these studies suggest that placental glycine production makes an important contribution to fetal glycine supply. In addition, we have recently shown (6) striking differences in the rate of transplacental transport of glycine vs leucine across the ovine placenta studied in vivo under chronic unstressed conditions. Glycine transport was equal to $<5 \%$ of the leucine transport over the first 90 min after a maternal bolus infusion of ${ }^{13} \mathrm{C}$-labeled glycine and leucine.
With the advent of FBS which permits sampling under relatively normal, unstressed conditions $(8-10,14)$ it is now possible to study the fetal enrichments and tracer concentrations of amino acids relative to the maternal enrichments and concentrations. Such data will be a complex function of transport and metabolism. There are always difficulties in attempting to assess organ function in vivo; data cannot be obtained in humans which describes the actual concentrations which exist at the maternal and fetal surfaces of the trophoblast. However, for other organs such as the liver, lung, and kidney, indirect indices of function have proven clinically useful. This study represents an attempt to explore whether this approach will prove useful for the human placenta. As a first attempt to analyze differences in the placental transport of glycine and leucine in vivo, we have explored the possibility that a bolus of ${ }^{13} \mathrm{C}$-labeled amino acids immediately preceding FBS might permit an estimate of their relative rates of transport. That hypothesis did not appear unreasonable given the fact that in ovine pregnancy difference between the maternal and fetal circulation are quite large and would have been detected for leucine and glycine regardless of which vessels within the two circulations were sampled, that is that arterial venous differences within the two circulations were always less than the difference between the maternal and fetal circulations. Figure 1 illustrates the fact that the fetal/maternal enrichment ratio of each amino acid changes over the first $300 \mathrm{~s}$ of study, whereas after this time it is relatively independent of time. In contrast, the ratio of glycine/leucine is unchanged from 40 to $1000 \mathrm{~s}$. The early part of the curve in Figure 1 reflects primarily placental permeability since there is less recirculation of tracer within the fetal circulation compared to the later time points. The latter part of the curve $(>300 \mathrm{~s})$ reflects both placental permeability and fetal and placental metabolism of glycine and leucine. For ethical reasons, we have used maternal venous samples rather than catheterizing the maternal arterial circulation for the sequential maternal samples required. There was also the practical clinical consideration that if we want to device an approach which could be used in high risk pregnancies, venous sampling would have major advantages. One can consider what the impact of sampling venous blood had upon the $\mathrm{F} / \mathrm{M}$ enrichments ratio. Inasmuch as venous enrichments would be less than arterial, the $\mathrm{F} / \mathrm{M}$ ratio is increased compared with arterial. Thus any differences found in the present study between leucine and glycine would have been even greater in using arterial enrichments.

The present study clearly demonstrates that in human pregnancy the appearance of $\left[{ }^{13} \mathrm{C}\right]$ glycine in the fetal circulation after a bolus in the maternal circulation is much slower than that of leucine, a difference which was also seen during in vivo studies of the ovine placenta. This difference could be due to any of several factors: 1 ) differences in placental permeability at the level of the trophoblast, presumably reflecting differences in affinity, concentration, etc., of the membrane-bound protein transporter; 2) differences in placental permeability at the level of the endothelium; or 3) differences in the rates of catabolism and production within the fetus and placenta. This latter possibility is likely to play some role since in vivo studies 
across the ovine placenta have demonstrated placental glycine production (21).

The data support the hypothesis that the human placenta is more permeable to leucine and glycine than is the epitheliochorial placenta of the sheep. In comparable studies in sheep, virtually no glycine enrichment was detectable in the fetal circulation.

The current approach provides considerable encouragement that it may now be possible to characterize relative rates of transport of amino acids across the human placenta under normal in vivo conditions. Such information can provide a foundation for an in vivo assessment of placental function.

\section{REFERENCES}

1. Kudo Y, Yamada K, Fujiwara A, Kawasaki T 1987 Characterization of amino acid transport systems in human placental brush-border membrane vesicles. Biochem Biophys Acta 904:309-318

2. Kudo Y, Boyd CAR 1990 Characterization of amino acid transport systems in human placental basal membrane vesicles. Biochem Biophys Acta 1021:169-174

3. Schneider H, Mohlen KH, Challier JC, Dancis J 1979 Transfer of glutamic acid across the human placenta perfused in vitro. Br J Obstet Gynaecol 86:299-306

4. Schneider H, Proegler M, Sodha R, Dancis J 1987 Asymmetrical transfer of $\alpha$-aminoisobutyric acid (AIB) leucine and lysine across in the vitro perfused human placenta. Placenta 8:141-51

5. Schneider H, Mohlen KH, Dancis J 1979 Transfer of amino acids across the in vitro perfused human placenta. Pediatr Res 13:236-240

6. Geddie S, Quick, AN, Meschia G, Wilkening RB, Fennessey PV, Battaglia FC 1992 Study of the transport of amino acids across the sheep placenta during non-steady state conditions. Pediatr Res 31:43A (abstr)

7. Bozzetti P, Ferrari MM, Marconi AM, Ferrazzi E, Pardi G, Makowski EL, Battaglia FC 1988 The relationship of maternal and fetal glucose concentrations in the human from mid gestation until term. Metabolism 37:358-363
8. Cetin I, Corbetta C, Sereni LP, Marconi AM, Bozzetti P, Pardi G, Battaglia FC 1990 Umbilical amino acid concentrations in normal and growth retarded fetuses sampled in utero by cordocentesis. Am J Obstet Gynecol 162:253-261

9. Marconi AM, Cetin I, Buscaglia M, Pardi G 1992 Mid gestation cord sampling: what have we learned? Placenta 13:115-122

10. Daffos F, Coppella-Pavlovsky M, Forestier F 1985 Fetal blood sampling during pregnancy with use of a needle guided by ultrasound: a study of 606 consecutive cases. Am J Obst Gynecol 153:665-660

11. Parazzini F, Cortinovis I, Bortolus R, Fedele L 1991 Standard di peso alle nascita in Italia. Ann Ostet Ginecol Med Perinat 112:203-246

12. Loy GL, Quick AN, Hay WW, Meschia G, Battaglia FC, Fennessey PV 1990 Feto-placental deamination and decarboxylation of leucine. Am J Physiol 259:E492E497

13. Cetin I, Sparks JW, Quick AN, Marconi AM, Meschia G, Battaglia FC, Fennessey PV 1991 Glycine turnover and oxidation and hepatic serine synthesis from glycine in fetal lambs. Am J Physiology 260:E371-E378

14. Pardi G, Cetin I, Marconi AM, Lanfranchi A, Bozzetti P, Ferrazzi E, Buscaglia M, Battaglia FC 1993 Diagnostic value of blood sampling in fetuses with growth retardation. N Engl J Med 328:692-696

15. Seber GAF, Wild CJ 1989. Nonlinear Regression. John Wiley \& Sons, New York, pp 191-270

16. vanVeen LCP, Meschia G, Hay WW, Battaglia FC 1987 Leucine disposal and oxidation rates in the fetal lamb. Metabolism 36:48-53

17. Kennaugh JM, Bell AW, Meschia G, Battaglia FC 1987 Ontogenetic changes in protein synthesis rate and leucine oxidation rate during fetal life. Pediatr Res 22:688-692

18. Marconi AM, Battaglia FC, Meschia G, Sparks JW 1989 A comparison of amino acid arteriovenous differences across the placenta and liver in the fetal lamb. Am J Physiol 257:E909-E915

19. Holtzman IR, Lemons JA, Meschia G, Battaglia FC 1979 Uterine uptake of amino acids and placental glutamine-glutamate balance in the pregnant ewe. J Dev Physiol 1:137-149

20. Morriss FH, Adcock III EW, Paxson CL, Greeley WJ 1979 Uterine uptake of amino acids throughout gestation in the unstressed ewe. Am J Obstet Gynecol 135:601-607

21. Cetin I, Fennessey PV, Sparks JW, Meschia G, Battaglia FC 1992 Fetal serine fluxes across the fetal liver, hindlimb and placenta in late gestation: the role of fetal serine in placenta glycine production. Am J Physiology 263:E786-E493 